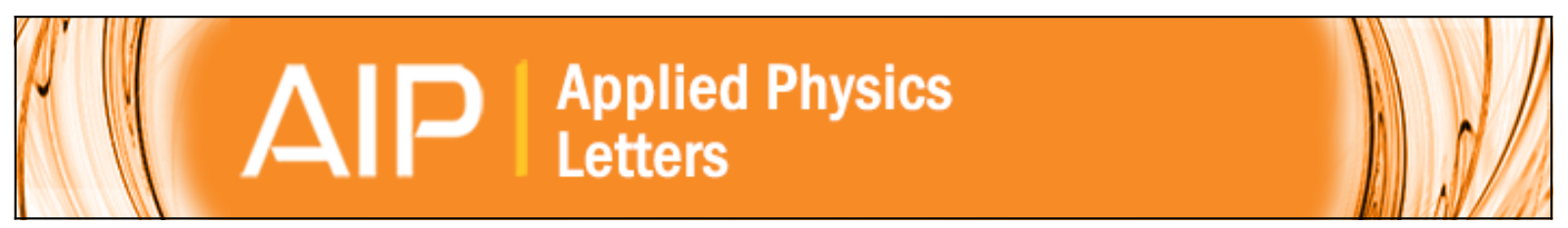

\title{
Side-dependent electron escape from graphene- and graphane-like SiC layers
}

Paola Gori, Olivia Pulci, Margherita Marsili, and Friedhelm Bechstedt

Citation: Applied Physics Letters 100, 043110 (2012); doi: 10.1063/1.3679175

View online: http://dx.doi.org/10.1063/1.3679175

View Table of Contents: http://scitation.aip.org/content/aip/journal/apl/100/4?ver=pdfcov

Published by the AIP Publishing

\section{Articles you may be interested in}

Communication: Electronic structure of the solvated chloride anion from first principles molecular dynamics J. Chem. Phys. 138, 181102 (2013); 10.1063/1.4804621

Electronic structures of graphane with vacancies and graphene adsorbed with fluorine atoms AlP Advances 2, 012173 (2012); 10.1063/1.3696883

Structural stability and electronic properties of $\mathrm{SiC}$ nanocones: First-principles calculations and symmetry considerations

Appl. Phys. Lett. 98, 123102 (2011); 10.1063/1.3567535

Calculated dependence of few-layer graphene on secondary electron emissions from SiC Appl. Phys. Lett. 98, 013109 (2011); 10.1063/1.3534805

A density functional theory-based study of the electronic structures and properties of cage like metal doped silicon clusters

J. Appl. Phys. 104, 084308 (2008); 10.1063/1.3000657

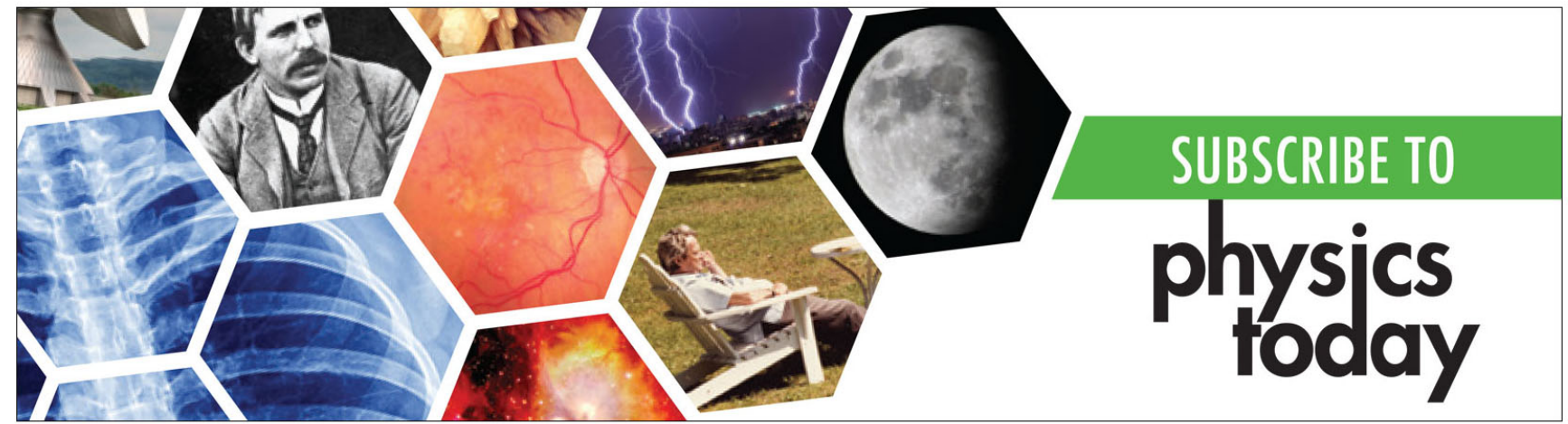




\title{
Side-dependent electron escape from graphene- and graphane-like SiC layers
}

\author{
Paola Gori, ${ }^{1, a)}$ Olivia Pulci, ${ }^{2}$ Margherita Marsili, ${ }^{2}$ and Friedhelm Bechstedt ${ }^{3}$ \\ ${ }^{1}$ ETSF, CNR-ISM, via Fosso del Cavaliere 100, 00133 Rome, Italy \\ ${ }^{2}$ ETSF, CNR-ISM, NAST, MIFP, Dipartimento di Fisica, Università di Roma "Tor Vergata," \\ Via della Ricerca Scientifica 1,00133 Rome, Italy \\ ${ }^{3}$ IFTO, Friedrich-Schiller-Universität and ETSF, Max-Wien-Platz 1, 07743 Jena, Germany
}

(Received 15 September 2011; accepted 31 December 2011; published online 26 January 2012)

\begin{abstract}
The structural and electronic properties of SiC-based two-dimensional (2D) crystals are studied by means of density functional theory and many-body perturbation theory. Such properties cannot simply be interpolated between graphene and silicene. The replacement of half of the $\mathrm{C}$ atoms by $\mathrm{Si}$ atoms opens a large direct electronic gap and destroys the Dirac cones. Hydrogenation further opens the gap and significantly reduces the electron affinity to 0.1 or $1.8 \mathrm{eV}$ in dependence on the carbon or silicon termination of the $2 \mathrm{D}$ crystal surface, thus showing a unique direction dependent ionization potential. This suggests the use of 2D-SiC:H as electron or hole filter. (C) 2012 American Institute of Physics. [doi:10.1063/1.3679175]
\end{abstract}

The recent discovery of the first two-dimensional (2D) crystalline nanomaterial, graphene, ${ }^{1}$ and its peculiar electronic properties lead to an explosion of scientific activity (see, e.g., review in Ref. 2). Structural or chemical modifications make the 2D carbon (C) allotrope an ideal material for nanotechnology. ${ }^{3}$ For instance, the fully hydrogenated graphene, an $s p^{3}$-bonded material called graphane, opens the fundamental gap from 0 to $5.4 \mathrm{eV} .^{4,5}$ The progress in preparation of graphene and its derivatives has generated interest for its hypothetical silicon ( $\mathrm{Si}$ ) counterparts, silicene, and silicane, i.e., a one-layer sheet of $\mathrm{Si}$ atoms arranged in a honeycomb lattice ${ }^{6}$ or in an $s p^{3}$-bonded hydrogen-passivated system. ${ }^{5}$ The existence of atomic layers of $s p^{2}$-bonded $\mathrm{Si}$ atoms is still a matter of controversy, but it has been recently shown to occur in silicene nanoribbons. ${ }^{7}$

Graphene planes can be prepared by $\mathrm{SiC}$ surface reconstruction under C-rich conditions. ${ }^{8}$ Fourfold-coordinated bulk $\mathrm{SiC}$ crystals exist in many polytypes which differ by the C-Si bilayer stacking. ${ }^{9}$ Playing with carbon and silicon at the nanoscale, ${ }^{10} \mathrm{SiC}$ nanotubes, ${ }^{11}$ and nanowires ${ }^{12}$ have been devised as promising candidates for hydrogen storage, nanoelectronics, and microelectromechanical systems. The SiCbased nanostructures are expected to allow the combination of ideas developed for carbon allotropes with the silicon technology. The stability of $\mathrm{SiC}$ nanotubes ${ }^{13}$ has suggested the possible existence of graphitic silicon carbide or, more interestingly, of corresponding graphene-like $\mathrm{SiC}$, the "silicongraphene." Total-energy calculations show the stability of such sheets. ${ }^{14}$ Hydrogenation of these 2D crystals may directly lead to graphane-like $\mathrm{SiC}$, the "silicongraphane" with $s p^{3}$-bonding.

In this letter, we study the structural, electrostatic, and electronic properties of 2D crystals made by pure and hydrogenated stoichiometric $\mathrm{SiC}$. We use density functional theory (DFT) in the generalized gradient approximation (GGA) for the geometry ${ }^{15,16}$ and Many-Body Perturbation Theory

\footnotetext{
${ }^{\text {a) }}$ Author to whom correspondence should be addressed. Electronic mail: paola.gori@ism.cnr.it.
}

within the GW approximation for the electronic quasiparticle (QP) band structure. ${ }^{17,18}$

The total-energy optimization of silicongraphene yields a flat honeycomb structure with two threefold coordinated atoms, $\mathrm{C}$ and $\mathrm{Si}$, in the hexagonal unit cell (Fig. 1(a)) similar to graphene and at odd with silicene, whose stable configuration is buckled. For the $s p^{2}+p_{z}$-bonded crystal silicongraphene, we find a bond length $d_{\mathrm{C}-\mathrm{Si}}=1.79 \AA$, that is intermediate between the $\mathrm{C}-\mathrm{C}$ bond length in graphene of $1.42 \AA$ and the Si-Si distance in silicene of $2.28 \AA$. The hydrogenation of each $\mathrm{C}$ and $\mathrm{Si}$ atom, keeping the $2 \mathrm{D}$ translational symmetry, leads to the silicongraphane geometry in Fig. 1(b). All atoms are fourfold coordinated and, hence, tend to be $s p^{3}$-hybridized. There are two consequences: (i) the silicongraphane sheet is slightly buckled, with a relative displacement of $\mathrm{C}$ and $\mathrm{Si}$ atoms along the sheet normal $\Delta=0.58 \AA$, in between that of graphane $(0.46 \AA)$ and of silicane $(0.72 \AA)$. (ii) All bonds are stretched. The length $d_{\mathrm{C}-\mathrm{Si}}=1.9 \AA$ is known from the bulk SiC polytypes. The corresponding values for the homomaterial $2 \mathrm{D}$ crystals are $d_{\mathrm{C}-\mathrm{C}}=1.54 \AA$ (graphane) and $d_{\mathrm{Si}-\mathrm{Si}}=2.36 \AA$ (silicane). An important hint for the stability of free-standing $\mathrm{SiC}$ sheets is given by their excess energy $E_{\text {exc }}(2 \mathrm{D}-\mathrm{SiC})=\left[E_{\mathrm{tot}}(2 \mathrm{D}-\mathrm{Si})\right.$ $\left.+E_{\mathrm{tot}}(2 \mathrm{D}-\mathrm{C})\right] / 2-E_{\mathrm{tot}}(2 \mathrm{D}-\mathrm{SiC})$, where $E_{\mathrm{tot}}$ is the total ground-state energy per unit cell of the sheets. They amount
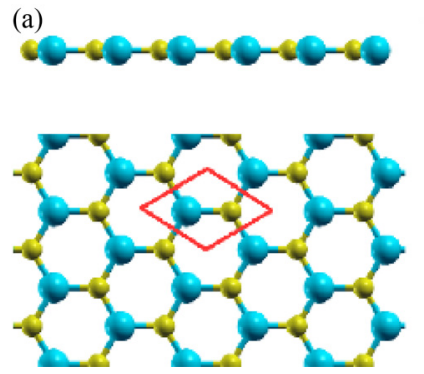

(b)
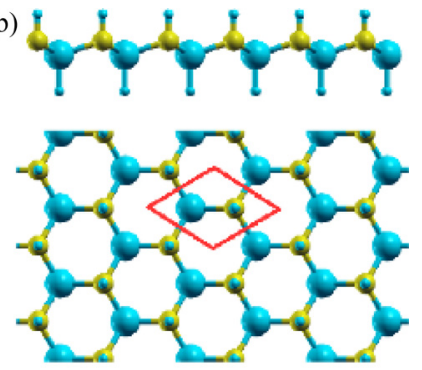

FIG. 1. (Color online) Side (upper panel) and top (lower panel) view of silicongraphene (a) and silicongraphane (b). Large (large blue) circles represent $\mathrm{Si}$ atoms, medium-sized (yellow) circles represent $\mathrm{C}$ atoms, while small (small blue) circles stand for $\mathrm{H}$ atoms. The unit cell is highlighted. 
to $E_{\mathrm{exc}}=-0.02 \mathrm{eV}$ for silicongraphene and $E_{\mathrm{exc}}=0.28 \mathrm{eV}$ for silicongraphane: in the absence of hydrogen, there is no gain in forming the $\mathrm{SiC}$ sheet with respect to graphene and silicene, while hydrogenation makes silicongraphane favorable.

Since silicane is a theoretically predicted but not yet realized material, it may be useful to analyze the practical realizability of $2 \mathrm{D}$ hydrogenated $\mathrm{SiC}$ by starting from a $\mathrm{SiC}$ surface. We consider a slab of hydrogenated C-terminated $3 \mathrm{C}-\mathrm{SiC}(111)$ and calculate the energy difference $\left[E_{\text {tot }}\right.$ $\left.\left(\mathrm{SiC}: \mathrm{H}_{5 \text { bil_slab }}\right)+E_{\text {tot }}(2 \mathrm{D}-\mathrm{SiC}: \mathrm{H})\right]-\left[E_{\text {tot }}\left(\mathrm{SiC}: \mathrm{H}_{6 \text { bil_slab }}\right)+E_{\text {tot }}\right.$ $\left.\left(\mathrm{H}_{2}\right)\right]=-0.15 \mathrm{eV}$, where $E_{\text {tot }}\left(\mathrm{SiC}: \mathrm{H}_{5(6) b i l \_s l a b}\right)$ denotes the total energy of a 5 (6)-bilayer $1 \times 1 \mathrm{SiC}(111)$ slab and $E_{\text {tot }}\left(\mathrm{H}_{2}\right)$ is the total energy of an hydrogen molecule. This suggests that an hydrogenated slab of $3 \mathrm{C}-\mathrm{SiC}(111)$ in presence of hydrogen can give rise to a stable 2D hydrogenated sheet of $\mathrm{SiC}$. Of course, a proper activation energy (not calculated here) should be considered for the process to take place. The dynamical stability of $2 \mathrm{D}-\mathrm{SiC}: \mathrm{H}$ has been theoretically investigated in a recent paper by calculating the phonon spectrum of the system. ${ }^{19}$ This further supports the feasibility of 2D-SiC:H.

The QP band structures versus high-symmetry lines in the hexagonal Brillouin Zone (BZ) for silicongraphene and silicongraphane are depicted in Fig. 2. The silicongraphene band structure is significantly changed with respect to graphene and silicene. ${ }^{20,21}$ Dramatic changes appear near the $K$ points for both $p_{z^{-}}$and $s p^{2}$-derived bands. The $p_{z}$-derived Dirac cones present in the graphene and silicene band structures are completely removed. Due to the ionic character of the bonds, instead, a gap is opened. Interestingly, as a remnant of the zero gap at $K$ in graphene and silicene, the $\mathrm{SiC}$ sheet still represents a direct semiconductor, although with a very large gap value of $3.7 \mathrm{eV}$ which is only slightly smaller than the indirect $K \rightarrow M$ gap (difference of $0.05 \mathrm{eV}$ ). Without QP corrections, i.e., within DFT-GGA, silicongraphene is an indirect semiconductor, although the two gaps $K \rightarrow M$ $(2.56 \mathrm{eV})$ and $K \rightarrow K(2.59 \mathrm{eV})$ are very close. Our QP gap corrections are of the order of $1.1-1.3 \mathrm{eV}$, in agreement with recent calculations. ${ }^{22}$

The hydrogenation of the $\mathrm{SiC}$ sheet with the $s p^{3}$ hybridization of the group-IV atoms changes significantly the band structure in Fig. 2(b). The disappearance of $p_{z}$-derived states leads to a further large opening of the gaps at $K$ and $M$. The sheet material is a direct semiconductor with a QP gap at $\Gamma$ of $5.3 \mathrm{eV}$. The QP effects are of the same order of magnitude as in the non-hydrogenated case. Qualitatively, the QP band structures are similar to those of graphane and silicane. ${ }^{5,23}$ Surprisingly, the fundamental gap of $2 \mathrm{D}-\mathrm{SiC}: \mathrm{H}$ is not close to an average of the pure systems, but it approaches the value of $5.4 \mathrm{eV}$ found for graphane (for silicane, the gap is $3.6 \mathrm{eV}$ ). This can be understood by the fact that in $2 \mathrm{D}-\mathrm{SiC}: \mathrm{H}$, the highest valence band is related to $\mathrm{C}$ atoms, while the lowest conduction band to hydrogen atoms bonded to the $\mathrm{C}$ atoms; hence, at least locally near the gap, the main electronic character is more "graphane-like" than "silicane-like."

Both crystals, 2D-SiC and 2D-SiC:H, show levels in the lower part of the conduction-band region which are built by rather delocalized nearly free electron (NFE)-like states. ${ }^{24}$ The second empty band in $\mathrm{SiC}$ possesses such a NFE character. In the case of $2 \mathrm{D}-\mathrm{SiC}: \mathrm{H}$, the NFE states become more important. They form the lowest conduction band. The character of the NFE states is clearly visible in the inset of Fig. 2(b). Noticeably, the maxima of the probability to find an electron only occur at the carbon-terminated side of a $\mathrm{SiC}: \mathrm{H}$ sheet.

The findings for the electronic states, especially regarding their distribution, have consequences for the electrostatics and the escape of electrons. In the case of silicongraphene in Fig. 2(a), the vacuum level $\varepsilon_{\text {vac }}$ appears $5.9 \mathrm{eV}$ above the valence-band maximum at $K$, just between the lowest $p_{z}$ and the second-lowest NFE conduction bands. The large ionization energy of $I=5.9 \mathrm{eV}$ correlates with the small electron affinity of $A=2.2 \mathrm{eV}$. The electron affinity is significantly lower than the one of the pure graphene and silicene, for both of which $A$ is larger than $4 \mathrm{eV}{ }^{5}$

In the case of $2 \mathrm{D}-\mathrm{SiC}: \mathrm{H}$, the asymmetry has a twofold effect (see Fig. 3): (i) First, two vacuum levels appear, a lower one in the range of the NFE band and a higher level in the region of the lowest empty $s p^{3}$ bands (cf. Fig. 2(b)). (ii) Especially the lower level is related to a significant reduction of the electron affinity to $A=0.1 \mathrm{eV}$, comparable with the value of $0.3 \mathrm{eV}$ found for pure graphane. ${ }^{5}$ The appearance of
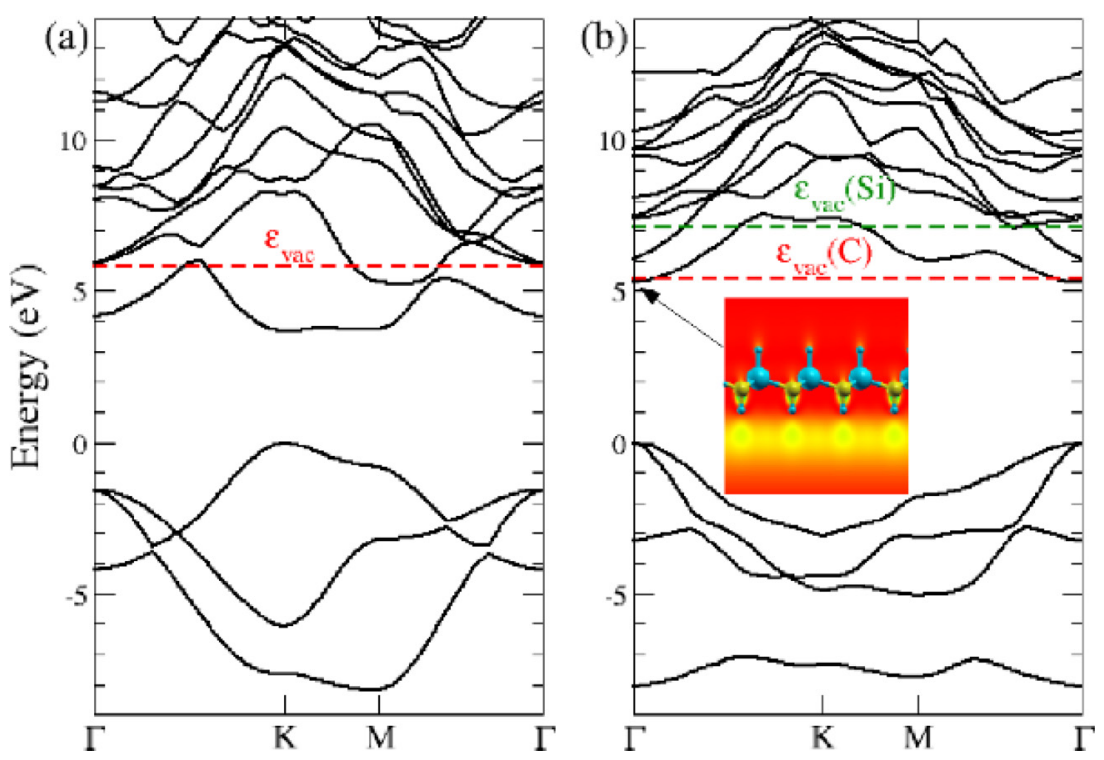

FIG. 2. (Color online) QP band structure of silicongraphene (a) and silicongraphane (b). The top of the valence bands is used as energy zero. The dashed horizontal lines indicate the position of the vacuum level(s). The inset shows the wave function at $\Gamma$ for the lowest conduction band. 
two vacuum levels is a consequence of the sheet polarity, i.e., the existence of a C-terminated and a Si-terminated side of the $\mathrm{SiC}: \mathrm{H}$ sheet. Their different properties are determined by the different electronegativity of $\mathrm{Si}, \mathrm{C}$, and $\mathrm{H}$. Figure 3 shows the presence of two vacuum levels by the planeaveraged electrostatic potential $V(z)$ through the unit cell of the artificial superlattice used for the modelling of the 2D$\mathrm{SiC}: \mathrm{H}$ crystal. Between the wells in Fig. 3 representing the sheets, the potential $V(z)$ exhibits two plateaus which can be identified with the two vacuum levels. The lower (upper) one is related to the $\mathrm{C}-\mathrm{H}(\mathrm{Si}-\mathrm{H})$ side in the supercell. Going through a SiC:H layer, a dipole discontinuity of about $1.7 \mathrm{eV}$ occurs. This potential step is due to the sheet buckling $\Delta$ and an effective electron transfer of $Q e$ from the $\mathrm{Si}$ to the $\mathrm{C}$ atoms but modified by the presence of partially ionized $\mathrm{H}$ atoms. The effective electron transfer between $\mathrm{Si}$ and $\mathrm{C}$ gives rise to a static dipole moment $Q e \Delta$ parallel to the sheet normal. More precisely, we have to study the electrostatics of a four-layer system of differently charged ions, $\mathrm{H}^{-\alpha}, \mathrm{Si}^{+\beta}$, $C^{-\gamma}, H^{+\delta}$, which is too complex. According to Gauss law, the discontinuity in the electrostatic potential in Fig. 3 can effectively be given by $\Delta V=\frac{4 \pi Q e}{\varepsilon_{s} A_{0}} \Delta$, where $\varepsilon_{s}=2.03$ is the static electronic dielectric constant of the $2 \mathrm{D}-\mathrm{SiC}: \mathrm{H}$ sheet arrangement (calculated within an independent-particle approximation), and $A_{0}=8.48 \AA^{2}$ is the area of a lateral unit cell containing one $\mathrm{C}$, one $\mathrm{Si}$, and two $\mathrm{H}$ atoms. This leads to $Q / \varepsilon_{s}=0.137$ and finally to $Q=0.252$, a much smaller value than expected from pure ionic bonding arguments and, hence, indicating a significant covalent bonding contribution to stabilize the $\mathrm{SiC}: \mathrm{H}$ sheet. The $Q$ value is also smaller than in bulk $\mathrm{SiC}$.

The existence of the potential discontinuity through the 2D crystal sheet has profound implications for the modification of the electron escape on both sides of the $\mathrm{SiC}: \mathrm{H}$ sheet. Due to the two vacuum levels, the ionization energy (electron affinity) of the C-terminated side $I_{\mathrm{C}}=5.4 \mathrm{eV}$ $\left(A_{\mathrm{C}}=0.1 \mathrm{eV}\right)$ is by about $1.7 \mathrm{eV}$ smaller than the corresponding value at the Si-terminated side, $I_{\mathrm{Si}}=7.1 \mathrm{eV}$ $\left(A_{\mathrm{Si}}=1.8 \mathrm{eV}\right)$. The differences do not mean that an energy

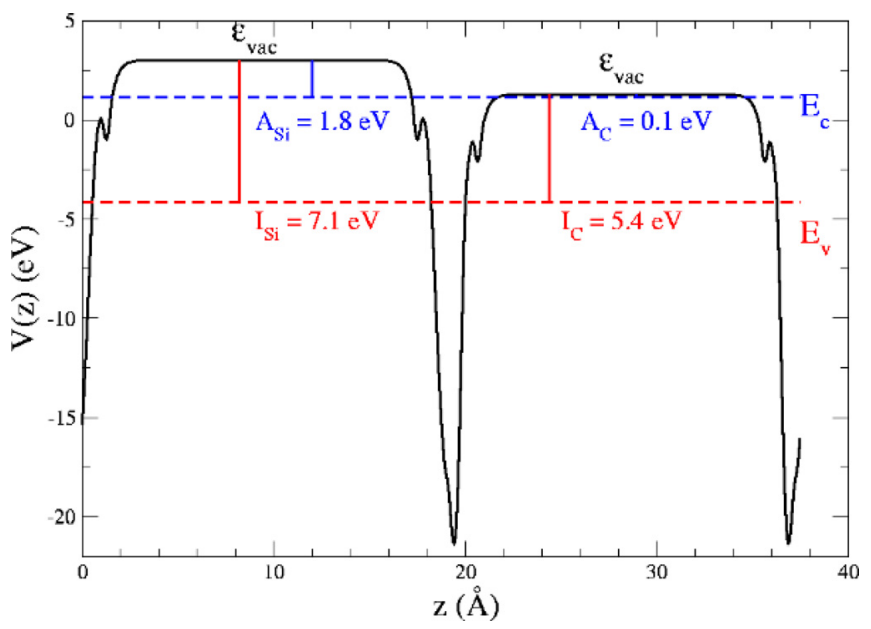

FIG. 3. (Color online) Electrostatic potential $V(z)$ averaged over planes parallel to the sheet versus the distance $z$ parallel to the sheet normal for silicongraphane. The two horizontal dashed lines indicate the energies of the lowest empty state and highest occupied state forming the fundamental QP gap. gain and hence a perpetuum mobile is possible by emitting electrons from the C-terminated side and reabsorbing these electrons at the Si-terminated side. The electron has to work against the dipole field. ${ }^{25}$ Nevertheless, we emphasize that the presence of an intrinsic dipole layer may have important implications for electronic, optoelectronic, or photovoltaic devices in which such $\mathrm{SiC}: \mathrm{H}$ sheet crystals are used to form heterostructures. In heterojunctions with one-and-the-same (but also two different) material(s) on both sides, the band offsets of electrons and holes can be tailored by the amount of the dipole potential $\Delta V$. In dependence on the orientation of the 2D crystal, i.e., its polarity, such a polar sheet may play the role of a barrier for electrons or holes. Consequently, electron or hole filters should be possible to fabricate by means of $\mathrm{SiC}: \mathrm{H}$ sheets. Interestingly, similar effects and applications of such dipole layers have been discussed for organic layers in organic light-emitting diodes and solar cells. $^{26,27}$

Summarizing, we have discussed the electronic properties of pure $s p^{2}$ - and $p_{z}$-bonded $\mathrm{SiC}$ sheets and their corresponding $s p^{3}$-bonded counterparts $\mathrm{SiC}: \mathrm{H}$ after hydrogenation. In silicongraphene, the ionic character of the $\mathrm{C}-\mathrm{Si}$ bonds opens the zero gap at the $K$ points of pure graphene or silicene. Hydrogenation further opens the gap with a minimum at BZ center $\Gamma$. The lower part of the conduction bands is determined by nearly free-electron states which allow a free electron motion in a plane parallel to the $\mathrm{C}$-side of the sheet crystal. The corrugation of the $\mathrm{SiC}$ sheet after hydrogenation yields a dipole layer. It leads to significant differences for the electron escape or capture in dependence on the C- or Si-termination of the layer side. This effect makes functionalized $\mathrm{SiC}$ layers very interesting for electronic applications, e.g., as electron or hole filters. The deposition of such 2D crystals may be possible on $\mathrm{SiC}$ and metal substrates using chemical vapor deposition and an appropriate precursor, e.g., monomethylsilane.

The authors thank Thomas Seyler for useful discussions. EU support through ETSF (GA 211956, user project no. 216), Clermont4 (GA 235114) and SIMTECH (GA 246937) projects is acknowledged. CPU time was granted by CINECA and ENEA-CRESCO.

${ }^{1}$ K. S. Novoselov, A. K. Geim, S. V. Morozov, D. Jiang, Y. Zhang, S. V. Dubonos, I. V. Grigorieva, and A. A. Firsov, Science 306, 666 (2004).

${ }^{2}$ A. H. Castro Neto, F. Guinea, N. M. R. Peres, K. S. Novoselov, and A. K. Geim, Rev. Mod. Phys. 81, 109 (2009).

${ }^{3}$ A. K. Geim, Science 324, 1530 (2009).

${ }^{4}$ S. Lebègue, M. Klintenberg, O. Eriksson, and M. I. Katsnelson, Phys. Rev. B 79, 245117 (2009).

${ }^{5}$ O. Pulci, P. Gori, M. Marsili, V. Garbuio, R. Del Sole, and F. Bechstedt, Strong excitons in novel two-dimensional crystals: Silicane and germanane (unpublished).

${ }^{6}$ G. G. Guzmán-Verri and L. C. Lew Yan Voon, Phys. Rev. B 76, 075131 (2007).

${ }^{7}$ P. De Padova, C. Quaresima, B. Olivieri, P. Perfetti, and G. Le Lay, Appl. Phys. Lett. 98, 081909 (2011).

${ }^{8}$ T. Ohta, A. Bostwick, T. Seyller, K. Horn, and E. Rotenberg, Science 313, 951 (2006).

${ }^{9}$ P. Käckell, B. Wenzien, and F. Bechstedt, Phys. Rev. B 50, 10761 (1994); 50, 17037 (1994).

${ }^{10}$ P. Melinon, B. Masenelli, F. Tournus, and A. Perez, Nature Mater. 6, 479 (2007).

${ }^{11}$ X.-H. Sun, C.-P. Li, W.-K. Wong, N.-B. Wong, C.-S. Lee, S.-T. Lee, and B.-K. Teo, J. Am. Chem. Soc. 124, 14464 (2002). 
${ }^{12}$ Z. Pan, H.-L. Lai, F. C. K. Au, X. Duan, W. Zhou, W. Shi, N. Wang, C.-S. Lee, N.-B. Wong, S.-T. Lee et al., Adv. Mater. 12, 1186 (2000).

${ }^{13}$ A. Mavrandonakis, G. E. Froudakis, M. Schnell, and M. Mülhäuser, Nano Lett. 3, 1481 (2003).

${ }^{14}$ H. Sahin, S. Cahangirov, M. Topsakal, E. Bekaroglu, E. Akturk, R. T. Senger, and S. Ciraci, Phys. Rev. B 80, 155453 (2009).

${ }^{15}$ J. P. Perdew, K. Burke, and M. Ernzerhof, Phys. Rev. Lett. 77, 3865 (1996).

${ }^{16}$ P. Giannozzi, S. Baroni, N. Bonini, M. Calandra, R. Car, C. Cavazzoni, D. Ceresoli, G. L. Chiarotti, M. Cococcioni, I. Dabo et al., J. Phys.: Condens. Matter 21, 395502 (2009)

${ }^{17}$ L. Hedin and B. J. Lundquist, in Solid State Physics, edited by H. Ehrereich, F. Seitz, and D. Turnbull (Academic, NY, 1969), Vol. 23, p. 1.

${ }^{18} \mathrm{QP}$ energies have been calculated with the non-self-consistent $\mathrm{GW}$ approximation, the $G_{0} W_{0}$ approach, using a plasmon pole model (A. Oschlies, R. W. Godby, and R. J. Needs, Phys. Rev. B 51, 1527 (1995)). We have used a $50 \times 50 \times 1 \mathrm{k}$ Monkhorst-Pack grid for the correlation part of the selfenergy, and $90 \times 90 \times 1 \mathrm{k}$ for the exchange part. The screening has been calculated using 300 bands. An artificial translational symmetry with a distance of $18.6 \AA$ between the sheets is used. In the hydrogenated case, the unit cell is doubled and includes two sheets, separated by $18.8 \AA$ with opposite dipole moments. The Coulomb potential has been cut in the $\mathrm{z}$ direction.

${ }^{19}$ X. Q. Wang and J. T. Wang, Phys. Lett. A 375, 2676 (2011).

${ }^{20}$ O. Pulci, P. Gori, M. Marsili, V. Garbuio, A. P. Seitsonen, F. Bechstedt, A. Cricenti, and R. Del Sole, Phys. Status Solidi A 207, 291 (2010).

${ }^{21}$ L. C. Lew Yan Voon, E. Sandberg, R. S. Aga, and A. A. Farajian, Appl. Phys. Lett. 97, 163114 (2010).

${ }^{22}$ E. Bekaroglu, M. Topsakal, S. Cahangirov, and S. Ciraci, Phys. Rev. B 81, 075433 (2010).

${ }^{23}$ M. Houssa, E. Scalise, K. Sankaran, G. Pourtois, V. V. Afanas'ev, and A. Stesmans, Appl. Phys. Lett 98, 223107 (2011).

${ }^{24}$ M. Posternak, A. Baldereschi, A. J. Freeman, E. Wimmer, and M. Weinert, Phys. Rev. Lett 50, 761 (1983).

${ }^{25}$ N. W. Ashcroft and N. D. Mermin, Solid State Physics (Saunders College, Philadelphia, 1976).

${ }^{26}$ G. Heimel, L. Romaner, J.-L. Bredas, and E. Zojer, Surf. Sci. 600, 4548 (2006).

${ }^{27}$ S. Duhm, G. Heimel, I. Salzmann, H. Glowatzki, R. L. Johnson, A. Vollmer, J. P. Rabe, and N. Koch, Nature Mater. 7, 326 (2008). 